\title{
A bijection for nonorientable general maps
}

\author{
Jérémie Bettinellil|
}

CNRS \& Laboratoire d'Informatique de l'École polytechnique, Palaiseau, France

\begin{abstract}
We give a different presentation of a recent bijection due to Chapuy and Dołęga for nonorientable bipartite quadrangulations and we extend it to the case of nonorientable general maps. This can be seen as a Bouttier-Di Francesco-Guitter-like generalization of the Cori-Vauquelin-Schaeffer bijection in the context of general nonorientable surfaces. In the particular case of triangulations, the encoding objects take a particularly simple form and we recover a famous asymptotic enumeration formula found by Gao.

Résumé. On donne une présentation différente d'une bijection récente due à Chapuy et Dołęga pour les quadrangulations biparties non-orientables et on l'étend au cas des cartes générales non-orientables. Cela peut se voir comme une généralisation à la Bouttier-Di Francesco-Guitter de la bijection de Cori-Vauquelin-Schaeffer dans le contexte des surfaces non-orientables générales. Dans le cas particulier des triangulations, les objets codant prennent une forme particulièrement simple et on retrouve la fameuse formule d'énumération asymptotique de Gao.
\end{abstract}

Keywords. map, graph, bijection, nonorientable surface, Brownian surface

\section{Introduction}

\subsection{Motivation}

The study of maps has seen tremendous developments in the past few decades. One of the reasons is that they provide natural discrete versions of a given surface. In particular, when taken according to a wellchosen natural probability distribution, it has been shown for several models that a random map converges (after scaling, in a certain sense) toward a limiting object. This limiting object is a random metric space and has (almost surely) the same topology as the surface on which the considered maps are drawn. It is called the Brownian map when the surface is the sphere, and the Brownian $\mathcal{S}$ for a general orientable surface $\mathcal{S}$.

In the most-studied case of the sphere, it has been shown [LG13, Mie13] for example that a uniform quadrangulation (map with only faces of degree 4 ) with $n$ faces converges to the Brownian map as $n \rightarrow \infty$. The case of a more general compact orientable surface (with a boundary allowed) has been studied, mostly in the context of uniform quadrangulations: partial convergence has been established in a series of papers ending with [Bet14] and a full convergence is under investigation [BM15b]. The full convergence in the particular case of the disk has recently been shown in [BM15a], where many more models are also considered.

\footnotetext{
†Email: jeremie.bettinellianormalesup.org.

Website: Www. normalesup.org/ bettinel
} 
All the previously mentioned results strongly rely on powerful bijective encodings of the considered maps. It turns out that quadrangulations are particularly well behaved with respect to these bijective encodings and this is the main reason why they are usually the first to be studied. However, if one wishes to study other models and, in particular, surfaces with a boundary, one needs more general bijective encodings. In the case of compact orientable surfaces, the so-called Schaeffer-like bijections [CV81, [Sch98, BDG04, CMS09, AB13] allow one to conduct most studies.

Until very recently, no such bijections were known in the case of a nonorientable surface. In [CD15], Chapuy and Dołęga took the first step by exhibiting a bijection allowing to encode nonorientable bipartite quadrangulations. In this work, we give an alternate description of their bijection, which provides an explicit construction for pointed quadrangulation $s^{(i)}$ and we show how to generalize it to nonorientable general maps. These works lay the bases for the future study of nonorientable Brownian surfaces [BCD15].

Another cause of interest for maps is their remarkable enumerative properties. In fact, although maps are intricate objects by nature, many classes of them possess a quite simple enumerative structure. Thanks to different involved enumeration techniques (generating functions, matrix integrals, algebraic combinatorics), many classes of maps have been enumerated and map enumeration has become over the years a full-fledged research domain.

In the case of the sphere, Tutte [Tut63] gave a very simple closed formula for the number of rooted maps with a given number of edges. A bijective proof of this formula was given by Cori and Vauquelin [CV81] and later popularized by Schaeffer [Sch98]. It relies on their so-called Cori-Vauquelin-Schaeffer bijection encoding quadrangulations of the sphere with trees whose vertices carry integer labels satisfying local constraints. For more general surfaces, Bender and Canfield [BC86] showed that the number of rooted maps with $n$ edges on a given surface (orientable or not) is asymptotically equal to a constant times $n^{5(h-1) / 2} 12^{n}$, where $h$ is the type of the considered surface and the constant depends on the surface. Extending the Cori-Vauquelin-Schaeffer bijection, a combinatorial interpretation of this fact in the orientable case was given by Chapuy, Marcus and Schaeffer [CMS09]. Their approach rely on a bijection between bipartite quadrangulations (it is a classical simple fact that bipartite quadrangulations are in bijection with general maps) and one-face maps of the same surface, whose vertices carry integer labels satisfying some local constraints.

In parallel, Bouttier, Di Francesco and Guitter [BDG04] extended the original Cori-Vauquelin-Schaeffer bijection to encode maps of the sphere with an arbitrary face degree distribution. Unifying both aforementioned extensions, Chapuy [Cha09] proved similar asymptotic enumeration results for more families of maps on an orientable surface.

Nonorientability does not causes too much difficulties for generating function approaches. In addition to Bender and Canfield's results, we may cite the work of Gao, who showed [Ga091] that the number of rooted triangulations with $n$ edges on a given surface (orientable or not) is asymptotically equal to a constant (depending on the surface) times $n^{5(h-1) / 2}(12 \sqrt{3})^{n}$. He also studied the algebraicity of the generating function of rooted maps on a given surface with face degree constraints [Ga093].

In their recent work [CD15], Chapuy and Dołęga extended the construction of [CMS09] to bipartite nonorientable quadrangulations. In this paper, we give a different construction of their bijection, and extend it by an approach reminiscent of [BDG04]. In order to achieve this goal, we somehow fix a local orientation of the surface via a global process, in the sense that the process uses the information of the

\footnotetext{
(i) A pointed map is a map given with a distinguished vertex. From a combinatorial point of view, it might not seem to make much difference, but the bijections for pointed maps turn out to be better behaved for probabilistic applications.
} 
whole map. As a result, the constraints satisfied by the labels of the encoding objects are also global and this provides us from giving a simple characterization of these objects. In the very particular case of quadrangulations, the global constraints can be expressed as local constraints and the encoding objects (so called well-labeled unicellular maps) take a simple form and this allowed Chapuy and Dołęga [CD15] to give a combinatorial interpretation of Bender and Canfield's asymptotic formula. In the particular case of triangulations, the same miracle occurs and we are able to give a combinatorial interpretation of the results of [Ga091].

\subsection{First definitions}

From now on and until the end of the paper, we fix a compact surface without boundary $\mathcal{S}$, orientable or not. A map is a cellular embedding of a finite graph (possibly with multiple edges and loops) into $\mathcal{S}$, considered up to homeomorphisms. Cellular means that the faces of the map (the connected components of the complement of edges) are homeomorphic to 2-dimensional open disks. A corner is an angular sector determined by two consecutive half-edges incident to the same vertex and to the same face. The degree of a face is its number of corners. All the maps we consider are rooted, that is, given with a distinguished oriented corner called the root. The (unoriented) corner corresponding to the root will be called the root corner, the vertex incident to the root will be called the root vertex, the face containing the root will be called the root face and the edge incident to the root face that follows the root will be called the root edge. See Figure 1

We will use the following involution we call a root flip: from a map $\mathfrak{m}$, we define the root flipped map $\overline{\mathfrak{m}}$ by rerooting $\mathfrak{m}$ at the only oriented corner incident to the other extremity of the root edge and defining the same root edge. We use the classical notation $V(\mathfrak{m})$ to denote the vertex set of a map $\mathfrak{m}$ and we denote by $d_{\mathfrak{m}}$ the graph metric on $V(\mathfrak{m})$. A map is bipartite if its vertex set can be partitioned into two subsets such that every edge links a vertex from one subset to a vertex from the other subset.

\subsection{Aim of the paper}

In this work, we present a bijection between the set of pointed bipartite maps on $\mathcal{S}$ and pairs consisting of what we call a welllabeled unicellular mobile and a parameter $\varepsilon \in\{+,-\}$. A welllabeled unicellular mobile is a one-face map with green or white vertices whose white vertices carry positive integer labels satisfying some compatibility relations, which need more background to be properly stated (see Definition 2 for a rigorous definition).

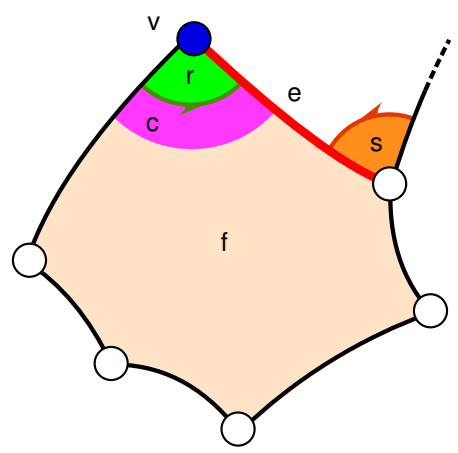

Fig. 1: The (green) root $\rho$, the (purple) root corner $c$, the (blue) root vertex $v$, the (light pink) root face $f$ and the (red) root edge $e$. The root of the root flipped map is $\sigma$ (in orange).

If the surface $\mathcal{S}$ is orientable, we recover the famous Bouttier-Di Francesco-Guitter bijection [BDG04] and the following basic properties continue to hold in the nonorientable case:

Proposition 1. If $\left(\mathfrak{m}, v^{\bullet}\right)$ is a pointed bipartite map and $((\mathfrak{u}, \mathfrak{l}), \varepsilon)$ denotes the corresponding pair, then

(i) $V(\mathfrak{m}) \backslash\left\{v^{\bullet}\right\}$ corresponds to the white vertices of $\mathfrak{u}$ and the label of a white vertex is given by its distance to $v^{\bullet}$ in $\mathfrak{m}$;

(ii) the faces of $\mathfrak{m}$ correspond to the green vertices of $\mathfrak{u}:$ moreover, the degree of a face of $\mathfrak{m}$ is twice the degree of the corresponding green vertex;

(iii) the maps $\mathfrak{m}$ and $\mathfrak{u}$ have the same number of edges. 
Property $(i)$ is absolutely crucial from a metric point of view, as the labeled unicellular map somehow captures part of the metric information of the map, namely all the distances to the distinguished vertex $v^{\bullet}$.

Our construction is based on a rule that gives an orientation to every corner of the map. We introduce what we call level loops; these can be thought of as contour lines in topography, where the height of a given vertex is its distance to the distinguished vertex $v^{\bullet}$. The orientation of the root gives a canonical orientation to all these level loops. Using these local orientations, we then apply similar rules as in the orientable case in order to complete the construction.

We will then see how to extend our bijection to general maps, which are not necessarily bipartite. In the case of triangulations, that is, maps with only faces of degree 3 , the encoding mobiles happen to have a particularly simple structure. This allows us to recover the following enumeration result ([Gao91, Theorem 1]).

Proposition 2. The number of (rooted) triangulations of $\mathcal{S}$ with $2 n$ faces (and thus $3 n$ edges and $n+2-2 h$ vertices, by the Euler characteristic formula) is asymptotically equivalent to

$$
c_{\mathcal{S}} n^{5(h-1) / 2}(12 \sqrt{3})^{n},
$$

where $h$ is the type of $\mathcal{S}$ and $c_{\mathcal{S}}$ is a constant that depends on $\mathcal{S}$.

The values for $h \leq 1$ of $c_{\mathcal{S}}$ are given in the full version [Bet15] of this paper. For small values of $h$, the generating function of triangulations can also be computed: we recover [Ga091, Theorem 3] and we add the case of the Klein bottle.

Proposition 3. The generating function of triangulations counted with weight $x$ per vertex is given by

$$
\begin{gathered}
\frac{1}{2}(1-2 \sigma)\left(1-\sigma+\sigma^{2}\right)-\frac{1}{2} \sqrt{1-6 \sigma+6 \sigma^{2}} \quad \text { if } \mathcal{S} \text { is the projective plane and } \\
3 \sigma(1-\sigma)\left(1-6 \sigma+6 \sigma^{2}\right)^{-2}\left(7-30 \sigma+30 \sigma^{2}-6(1-2 \sigma) \sqrt{1-6 \sigma+6 \sigma^{2}}\right)
\end{gathered}
$$

if $\mathcal{S}$ is the Klein bottle, where $\sigma$ is an algebraic function of $x$ given by

$$
x=\frac{1}{2} \sigma(1-\sigma)(1-2 \sigma) \quad \sigma(0)=0 .
$$

The analog of Proposition 2 was obtained by the same method for quadrangulations on an orientable surface in [CMS09], as well as on a nonorientable surface in [CD15]. Unfortunately, the constraints on the labels of a well-labeled unicellular mobile in general are too intricate to derive similar enumeration results. In fact, the labels satisfy global constraints instead of just local constraints as soon as the maps we consider are neither triangulations nor quadrangulations.

\section{From pointed bipartite maps to well-labeled unicellular mobiles}

Let $\left(\mathfrak{m}, v^{\bullet}\right)$ be a pointed bipartite map. For every $v \in V(\mathfrak{m})$, we define its label by $\mathfrak{l}(v):=d_{\mathfrak{m}}\left(v^{\bullet}, v\right)$. The fact that $\mathfrak{m}$ is bipartite implies that the labels of two neighboring vertices differ by exactly 1 . If the root vertex has a smaller label than the other extremity of the root edge, we set $\varepsilon:=+$; otherwise, we replace $\mathfrak{m}$ by its root flipped version $\overline{\mathfrak{m}}$ and $\operatorname{set} \varepsilon:=-$. We construct the level loops as follows. At first, we only construct unoriented level loops: we will orient all the loops at the end. We consider the border 
of a face along some edge and we denote by $i-1$ and $i$ the labels of the vertices incident to this edge. We travel on the border of the face along the edge, from the vertex labeled $i-1$ toward the vertex labeled $i$. When we reach the vertex labeled $i$, we turn around the vertex until we see an edge linking it to a vertex with label $i-1$; in the process, we cross a nonnegative number of edges linking the vertex to vertices labeled $i+1$. We then travel along the edge toward the vertex labeled $i-1$. We keep traveling along the border of the face we are visiting until we either close the loop or reach a vertex labeled $i+1$. In the later case, the vertex visited just before the vertex labeled $i+1$ necessarily has label $i$; we turn around this vertex until we see an edge linking it to a vertex with label $i-1$. We iterate the process until we close the loop. We say that such a loop is at level $i$. We iterate the process until each side of every edge of type $i-1-i$ is visited by a loop at level $i$. We finally add around $v^{\bullet}$ a single loop at level 0 .

We orient, give an origin and order the level loops as follows. We consider all the loops visiting the root corner: note that there exists at least one such loop. We orient all these loops according to the orientation given by the root, we set their origin at the root corner and we declare them to be the first loops, ordered by increasing level. We then start from the origin of the first loop and travel on it. Every time we encounter new loops, we declare them to be the next ones, ordered in increasing level. We set their origin at the location we are and we orient them by the orientation induced by the loop on which we are traveling. When we arrive back at the origin of our loop, we move to the next one and iterate the process until every loop has been oriented. See Figure 3 . Note that this operation terminates as every loop at level $i \geq 1$ touches a loop at level $i-1$.

We consider a loop and denote by $i$ its level. The loop visits one by one several corners of the map. Among these corners, we say that the loop selects those with label $i$ that are immediately preceded by a corner labeled $i-1$. We add an extra vertex in the middle of every face: these vertices will be called green vertices in the following, in contrast with the original vertices, which we will call white vertices. Inside each face, we link in a noncrossing fashion by green edges all the corners that are selected by some loop to the green vertex of the face.

Finally, we consider the embedded graph $\mathfrak{u}$ whose vertex set consists of the union of $V_{\circ}(\mathfrak{u}):=V(\mathfrak{m}) \backslash\left\{v^{\bullet}\right\}$ with the set $V_{\bullet}(\mathfrak{u})$ of green vertices and whose edge set is composed of the green edges. We root it with the convention depicted on Figure 2, The

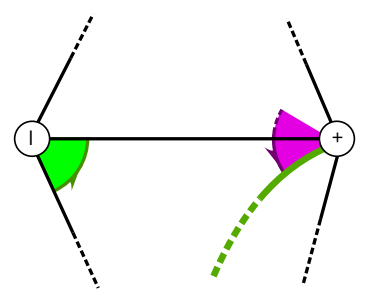

Fig. 2: Rooting $\mathfrak{u}$ from the root of $\mathfrak{m}$. The root of $\mathfrak{m}$ is represented in green and the root of $\mathfrak{u}$ is in purple. The existence of the green edge (of $\mathfrak{u}$ ) is ensured by the rules of the construction. white vertices of $\mathfrak{u}$ inherit the labels from the function $\mathfrak{l}$ : we set $\Phi\left(\mathfrak{m}, v^{\bullet}\right):=((\mathfrak{u}, \mathfrak{l}), \varepsilon)$. See Figure 4

\section{Definition of the encoding object and inverse construction}

\subsection{Preliminaries}

We now describe in more details the encoding object we obtained in Section 2 First, we say that a pair $(\mathfrak{u}, \mathfrak{l})$ is a labeled unicellular mobile if it satisfies the following conditions:

$\diamond \mathfrak{u}$ is a rooted one-face map of $\mathcal{S}$ whose vertex set is partitioned into $V_{\bullet}(\mathfrak{u}) \sqcup V_{\circ}(\mathfrak{u})$ in such a way that every edge links a vertex from $V_{\bullet}(\mathfrak{u})$ to a vertex from $V_{\circ}(\mathfrak{u})$; 

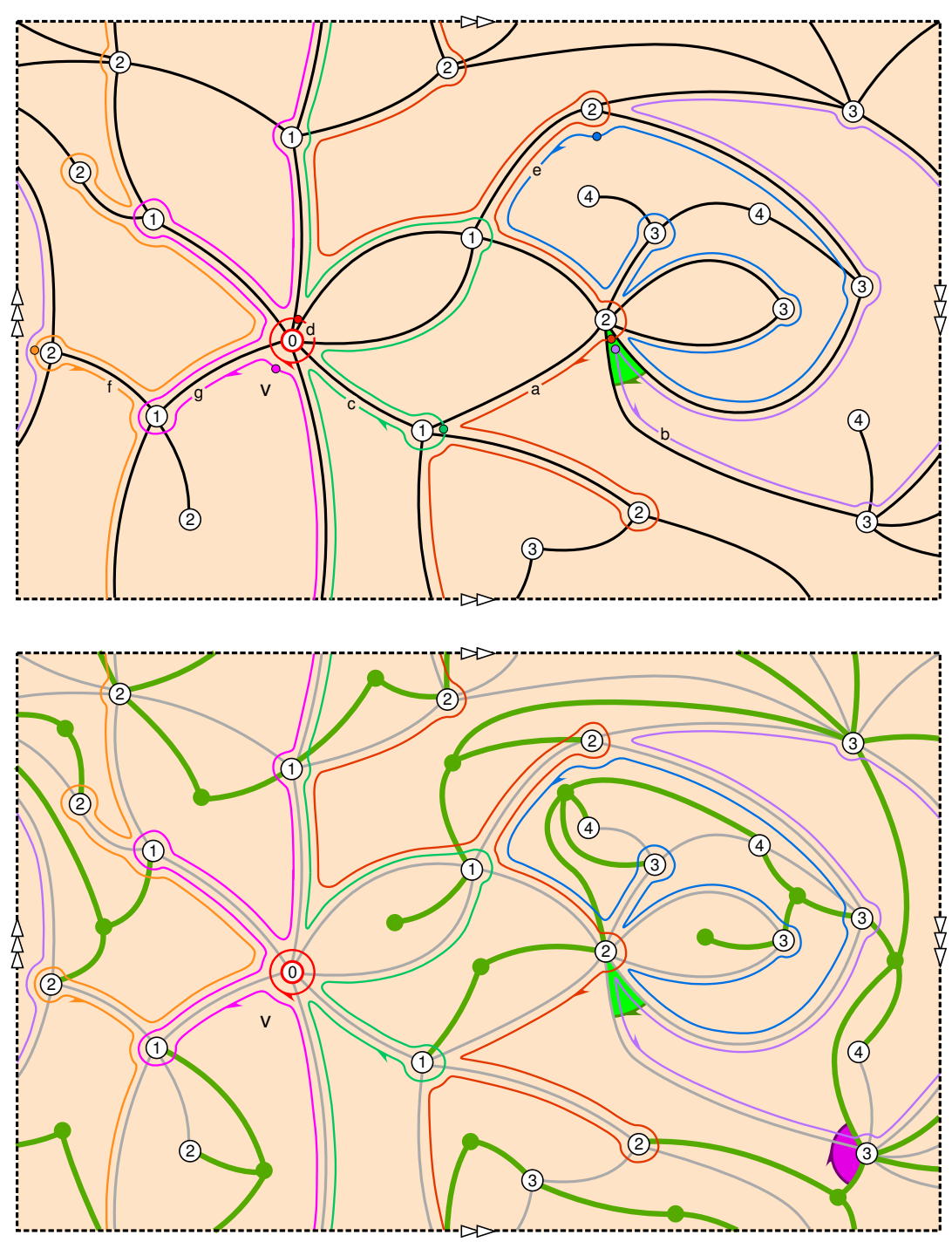

Fig. 3: The level loops. Inside each face, the single loop at maximal level is not represented and not taken into account in the ordering of the loops. The letters indicate the ranks of the loops in the order, the half-arrowheads their orientation and the dots their origin.

Fig. 4: The bijection, from a pointed bipartite map to a well-labeled unicellular mobile. The edges of the original map have been grayed out.

$\diamond \mathfrak{l}: V_{\circ}(\mathfrak{u}) \rightarrow \mathbb{N}$ is a function with minimum 1 ;

$\diamond$ the root vertex belongs to $V_{\circ}(\mathfrak{u})$.

As the face of $\mathfrak{u}$ is homeomorphic to a disk by definition, and as the root of $\mathfrak{u}$ gives a canonical orientation to this disk, the corners of $\mathfrak{u}$ are naturally ordered in a cyclic way around its face. In what follows, we only consider the corners of $\mathfrak{u}$ that are incident to vertices of $V_{\circ}(\mathfrak{u})$.

Definition 1. We call arc any contiguous interval of two or more subsequent corners such that the first and last corners have strictly smaller labels than the other corners. The first and last corners of the arc are called its extremities and the other corners are called its internal corners. 
An arc whose extremities have labels $i$ and $j$ is called an $\{i, j\}$-arc and its level is defined as the number $i \vee j$. An arc is said to be trivial if it contains only two corners.

The following lemma is quite straightforward so that, for space reasons, we give it without proof and refer the interested reader to the full version of the paper.

Lemma 4. We have the following properties.

(i) Let $A$ and $B$ be two distinct nontrivial arcs whose intersection contains internal corners of $A$ or $B$. Then $A \subseteq B$ or $B \subseteq A$ and the level of the larger arc is strictly lower than that of the smaller arc.

(ii) The following properties are equivalent:

(a) For all $i \geq 1$, every nontrivial arc at level $i$ contains a corner with label $i+1$.

(b) For all $i \geq 1$, every nontrivial arc at level $i$ has a range of internal corner labels of the form $\{i+1, i+2, \ldots, m\}$ for some $m$.

(c) For all $i \geq 2$ and for every corner with label $i$, either the first subsequent corner with label strictly smaller than $i$ has label $i-1$ or the last preceding corner with label strictly smaller than $i$ has label $i-1$.

(iii) The properties of (ii) imply that, for all $i \geq 2$, every arc at level $i \geq 2$ and every corner labeled $i \geq 2$ is included in a unique arc at level $i-1$, which is nontrivial.

\subsection{Construction}

In order to give the explicit conditions satisfied by a well-labeled unicellular mobile, we need to start performing the inverse construction. We suppose that $(\mathfrak{u}, \mathfrak{l})$ is a labeled unicellular mobile satisfying the properties of Lemma 4|(ii). We add inside the unique face of $\mathfrak{u}$ a new vertex $v^{\bullet}$ with label $\mathfrak{l}\left(v^{\bullet}\right):=0$. We connect all the corners with label 1 to $v^{\bullet}$ in a noncrossing fashion and, for each nontrivial arc at level $i \geq 2$, we add a temporary vertex to which we link all its corners that are labeled $i+1$. Lemma $4 \mid(i)$ ensures that this can be done in a noncrossing fashion.

We now construct the unoriented level loops. Notice that, by Lemma 4[(iii) every corner of $\mathfrak{u}$ is linked either to $v^{\bullet}$ or to a temporary vertex. We call black edges these links. We select a corner of $\mathfrak{u}$ and one incident edge of $\mathfrak{u}$. We denote by $i$ the label of the selected corner. Starting from the selected corner, we explore the boundary of the face of $\mathfrak{u}$, in the orientation given by the selected edge. We search the first subsequent corner with label smaller than or equal to $i$. We then begin to draw a loop from the selected corner to the second one, without crossing black edges; this can be done as two corners of $\mathfrak{u}$ are linked to the same temporary vertex if and only if they have the same label and all the corners in one of the two intervals they delimit have labels larger than or equal to this common label. We then cross the edge of $\mathfrak{u}$ at the second corner and iterate the process (always with the same $i$ ) until we close the loop. We thus create a loop and define its level to be $i$. We iterate the process until every side of every black edge starting from a corner labeled $i$ is visited by a loop at level $i$. We add a single loop at level 0 around $v^{\bullet}$. See Figure 5 .

By construction, every portion of loop included in the face of $\mathfrak{u}$ delimits an arc of $(\mathfrak{u}, \mathfrak{l})$. More precisely, every arc delimited by a portion of loop at level $i$ is either trivial at level less than or equal to $i$ or nontrivial at level $i$. Moreover, every nontrivial arc is delimited by exactly one loop and every trivial arc is delimited by at least one loop. We say that a level loop at level $i$ is well oriented if every nontrivial arc it delimits is such that the extremity first visited by the portion of loop inside the face of $u$ has label $i$. 


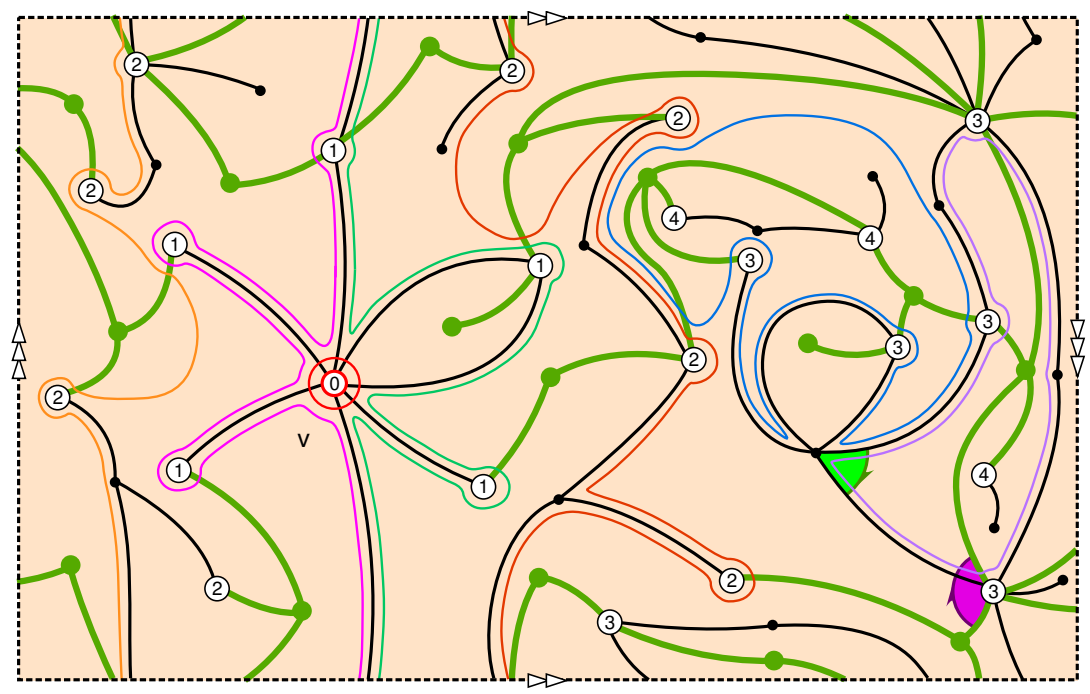

Fig. 5: The unoriented level loops. Here again, we did not represent the loops at maximal level, that is, the loops circling around a single green vertex.

Let $A$ be an arc at level $i \geq 2$ and let $B$ be the arc at level $i-1$ defined by Lemma 4(iii), As $B$ is nontrivial, there is a unique loop that delimits it. We say that this loop overflies the portions of loops delimiting $A$ (there may be more than one portion if $A$ is trivial). We also say that the portions of loops delimiting the arcs at level 1 are overflown by the single loop at level 0 . We similarly define the loop overflying a corner.

We define a future root as shown on Figure 6 We will orient, give an origin and order a few level loops. Every time we orient a loop, we do the following. If the loop is not well oriented, we stop the process. If the loop is well oriented, for each nontrivial arc it delimits, we identify the corresponding temporary vertex with the extremity of the arc that is first visited by the portion of loop inside the face of $\mathfrak{u}$. By Lemma $4[(i)$, this operation can be done in such a way that the black edges do not cross each other. See Figure 7.

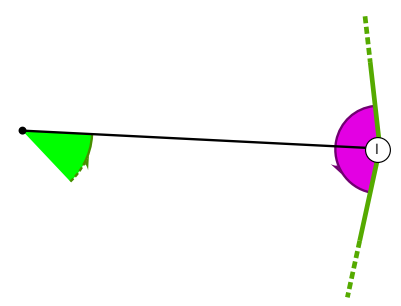

The first loop in our order is the loop overflying the root corner

Fig. 6: The future root of $\mathfrak{m}$ (in green) from the root of $\mathfrak{u}$ (in purple).

of $\mathfrak{u}$. We orient it by the orientation prescribed by the future root and do the aforementioned identifications (provided that it is well oriented). After these identifications, the first loop passes through the future root corner. We set its origin at this location. We then travel on the first loop, starting at its origin. Every time we see an unoriented loop, we orient it in the same orientation as the orientation of the loop on which we are traveling. We declare this loop to be the next one and we set its origin at the location we are. If we see several new loops at the same time, we order them by increasing level. When we arrive back at the origin of our loop, we move to the next one and iterate the process until either we create a loop that is not well oriented or every loop has been oriented. Observe that, as every portion of loop sees the loop that overflies it, this process terminates.

Definition 2. A well-labeled unicellular mobile is a labeled unicellular mobile that satisfies the properties of Lemma 4,(ii) and such that the previous process only creates well oriented level loops. 

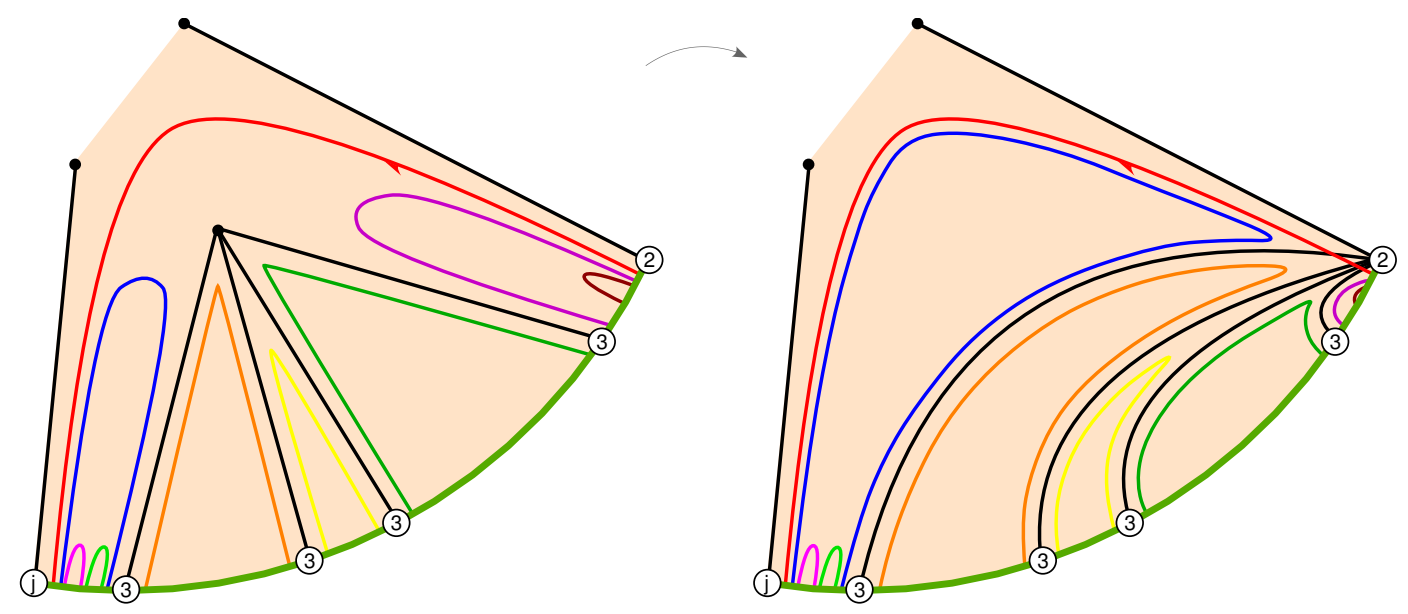

Fig. 7: Identification of a temporary vertex. The red loop delimits the $\{i, j\}$-arc $(i \geq j)$ and the temporary vertex corresponding to this arc is identified with the first extremity visited by the portion of loop. When traveling on the red loop, we "see" the other loops in the order brown, purple, green, yellow, orange, blue, then the overflying loop (not represented) and finally light green and light purple.

We now suppose that $(\mathfrak{u}, \mathfrak{l})$ is a well-labeled unicellular mobile and we perform the previous construction. We denote by $\mathfrak{m}$ the map whose vertex set is $V_{\circ}(\mathfrak{u}) \cup\left\{v^{\bullet}\right\}$, whose edges are the black edges and whose root is the future root. We also denote by $\overline{\mathfrak{m}}$ the root flipped version of $\mathfrak{m}$ and $\operatorname{set} \Psi((\mathfrak{u}, \mathfrak{l}),+):=\left(\mathfrak{m}, v^{\bullet}\right)$ and $\Psi((\mathfrak{u}, \mathfrak{l}),-):=\left(\overline{\mathfrak{m}}, v^{\bullet}\right)$. See Figure 8

\section{The previous mappings are inverse one from another}

We denote by $\mathcal{B}^{\bullet}$ the set of pointed bipartite maps of the surface $\mathcal{S}$ we consider and by $\mathcal{U}$ the set of well-labeled unicellular mobiles (defined in Section 3). The proof of the following theorem takes a few pages and can be found in the full version.

Theorem 5. The mappings $\Phi: \mathcal{B}^{\bullet} \rightarrow \mathcal{U} \times\{+,-\}$ and $\Psi: \mathcal{U} \times\{+,-\} \rightarrow \mathcal{B}^{\bullet}$ are inverse bijections.

Remark. If $\mathfrak{m}$ is a quadrangulation, by Proposition 1 (ii), all the vertices in $V_{\bullet}(\mathfrak{u})$ have degree 2 . We can remove them and merge the two incident edges into a single edge. Through this operation, $(\mathfrak{u}, \mathfrak{l})$ becomes a so-called well-labeled unicellular map and one recovers (a slight modification of) the Chapuy-Dołega bijection [CD15].

\section{General maps}

We now relax the hypothesis that the map is bipartite. We will slightly modify it in order to be able to apply our bijection from Section 2 We consider a general pointed map $\left(\mathfrak{m}, v^{\bullet}\right)$ and define the labeling function $\mathfrak{l}: V(\mathfrak{m}) \rightarrow \mathbb{Z}_{+}$as before. There are now two kinds of edges: an edge will be called equilabeled if its extremities both have the same label. We then enlarge the map $\mathfrak{m}$ by adding in the middle of each equilabeled edge an extra vertex splitting the edge into two new edges. We denote by $\tilde{\mathfrak{m}}$ this enlarged 


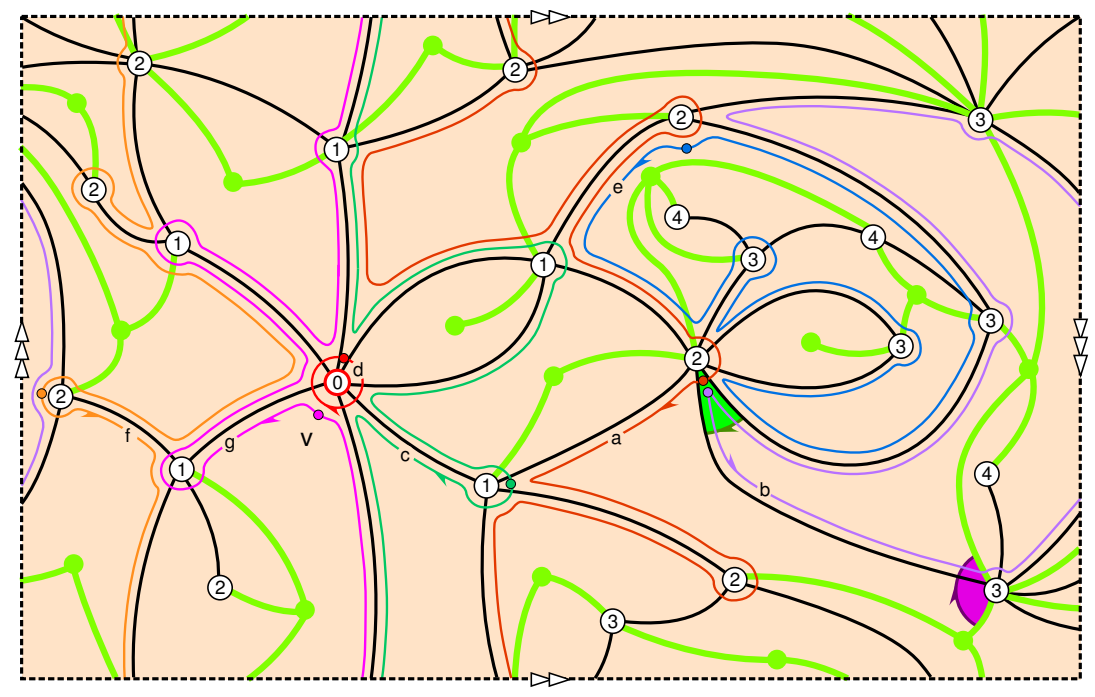

Fig. 8: The bijection, from a well-labeled unicellular mobile to a pointed bipartite map.

map and we assign to each added vertex the common label of its two neighbors plus 1 . This extends the definition of $\mathfrak{l}$ to $V(\tilde{\mathfrak{m}})$ and, clearly, for $v \in V(\tilde{\mathfrak{m}})$, one has $\mathfrak{l}(v)=d_{\tilde{\mathfrak{m}}}\left(v^{\bullet}, v\right)$.

The map $\tilde{\mathfrak{m}}$ is bipartite, so we may apply the construction of Section 2 . we set $((\tilde{\mathfrak{u}}, \mathfrak{l}), \varepsilon):=\Phi\left(\tilde{\mathfrak{m}}, v^{\bullet}\right)$. We slightly modify the encoding map as follows. Every vertex of $V(\tilde{\mathfrak{m}}) \backslash V(\mathfrak{m})$ is by design of degree 2 and both corners incident to it are selected by the level loops, as they correspond to local maximums along the boundaries of the incident faces. As a result, it also has degree 2 in $\tilde{\mathfrak{u}}$; we suppress it and merge the two incident edges into a single edge. We call such a resulting edge a flagged edge and we assign to it the label of the suppressed vertex. We denote by $(\mathfrak{u}, \mathfrak{l})$ the resulting map, the function $\mathfrak{l}$ being defined on a subset of the vertices and edges of $\mathfrak{u}$. Finally, if the root edge of $\mathfrak{m}$ is equilabeled, then the root vertex of $\tilde{\mathfrak{u}}$ is one of the added vertices of $\tilde{\mathfrak{m}}$. In this case, we transgress our usual definition of root and declare the root of $\mathfrak{u}$ to be the edge resulting of the suppression of the root vertex of $\tilde{u}$, together with the side and local orientation induced by the root of $\tilde{\mathfrak{u}}$. Such a map will be called edge-rooted in what follows. See Figure 9 . We extend the definition of $\Phi$ by setting $\Phi\left(\mathfrak{m}, v^{\bullet}\right):=((\mathfrak{u}, \mathfrak{l}), \varepsilon)$. The extended mapping $\Phi$ realizes a bijection between the set of pointed maps of $\mathcal{S}$ and its image. See the full version for the details.

\section{Application to triangulations}

In the case of triangulations, we can give a simpler characterization of the encoding objects and derive enumeration results. In fact, the green vertices of an encoding object can only be of the three types depicted on Figure 10.

Moreover, the label variations along two consecutive corners of a unicellular mobile whose green vertices are of such a type belong to $\{-1,0,1\}$. This entails that such a mobile satisfies the properties of Lemma $4($ ii $)$, that every of its nontrivial arcs has the same label at both extremities, and finally that every orientation of any of its level loops is a good orientation. Moreover, the conditions on the labels entail that both extremities of every nontrivial arc are original white vertices (in the sense that they do not come from vertices added on the flagged edges) so that no temporary vertices will be identified with vertices added on 


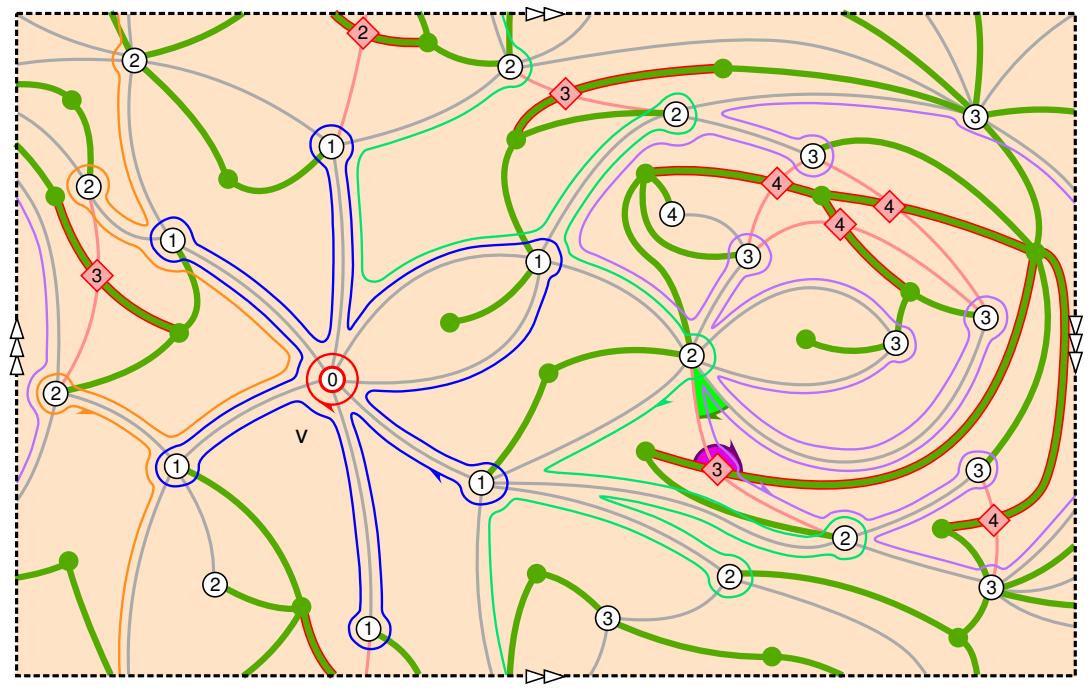

Fig. 9: The bijection for a general map. The flagged edges of the mobile have been highlighted and their labels are represented by the red squares. On this example, the root edge is equilabeled; the root of the mobile is thus a flagged edge given with a side and local orientation.
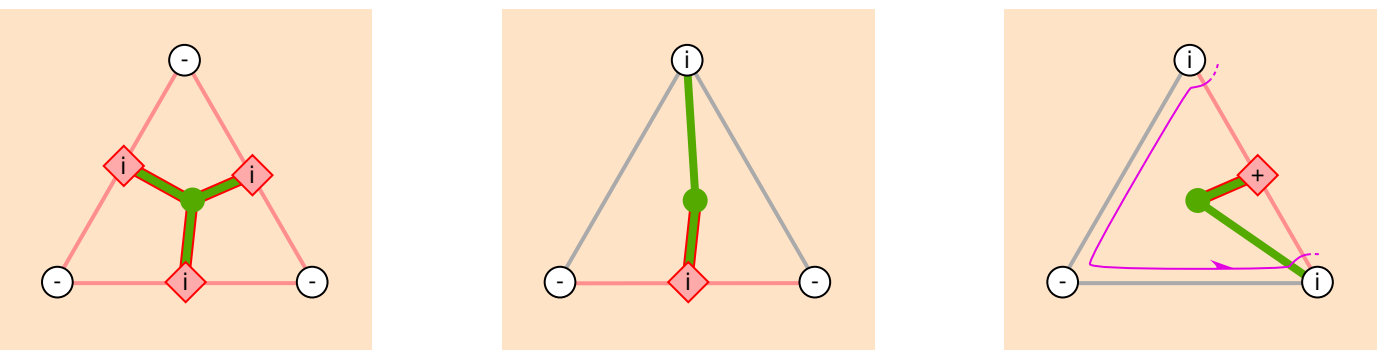

Fig. 10: The three possible types of face and the corresponding green vertices.

the flagged edges. As a result, the image by $\Phi$ of the set of pointed triangulations is exactly the set of these objects. They are simple enough to describe and can be enumerated by generating function techniques. We refer the reader to the full version [Bet15] for this application and the proof of Propositions 2 and 3

\section{Acknowledgements}

This work is partially supported by Grant ANR-14-CE25-0014 (GRAAL). The author also acknowledges partial support from the Isaac Newton Institute for Mathematical Sciences where part of this work was conducted. We thank Guillaume Chapuy and Maciej Dołęga for stimulating discussions and for sharing [CD15] with us, as well as Grégory Miermont for encouraging discussions, especially about further developments on nonorientable Brownian surfaces. 


\section{References}

[AB13] Ambjørn \& Budd. Trees and spatial topology change in causal dynamical triangulations. $J$. Phys. A, 46(31):315201, 33, 2013.

[BC86] Bender \& Canfield. The asymptotic number of rooted maps on a surface. J. Combin. Theory Ser. A, 43(2):244-257, 1986.

[BCD15] Bettinelli, Chapuy \& Dołęga. Nonorientable Brownian surfaces. In preparation, 2015.

[BDG04] Bouttier, Di Francesco \& Guitter. Planar maps as labeled mobiles. Electron. J. Combin., 11(1):Research Paper 69, 27 pp. (electronic), 2004.

[Bet14] Bettinelli. Geodesics in Brownian surfaces (Brownian maps). ArXiv:1401.3602, to appear in Ann. Inst. Henri Poincaré Probab. Stat., 2014.

[Bet15] Bettinelli. A bijection for nonorientable general maps. ArXiv:1512.02208, 2015.

[BM15a] Bettinelli \& Miermont. Compact Brownian surfaces I. Brownian disks. ArXiv:1507.08776, 2015.

[BM15b] Bettinelli \& Miermont. Compact Brownian surfaces II. The general case. In preparation, 2015.

[CD15] Chapuy \& Dołęga. A bijection for rooted maps on general surfaces. ArXiv:1501.06942, 2015.

[Cha09] Chapuy. Asymptotic enumeration of constellations and related families of maps on orientable surfaces. Combin. Probab. Comput., 18(4):477-516, 2009.

[CMS09] Chapuy, Marcus \& Schaeffer. A bijection for rooted maps on orientable surfaces. SIAM J. Discrete Math., 23(3):1587-1611, 2009.

[CV81] Cori \& Vauquelin. Planar maps are well labeled trees. Canad. J. Math., 33(5):1023-1042, 1981.

[LG13] Le Gall. Uniqueness and universality of the Brownian map. Ann. Probab., 41(4):2880-2960, 2013.

[Gao91] Gao. The number of rooted triangular maps on a surface. J. Combin. Theory Ser. B, 52(2):236249, 1991.

[Gao93] Gao. The number of degree restricted maps on general surfaces. Discrete Math., 123(1-3):4763, 1993.

[Mie13] Miermont. The Brownian map is the scaling limit of uniform random plane quadrangulations. Acta Math., 210(2):319-401, 2013.

[Sch98] Schaeffer. Conjugaison d'arbres et cartes combinatoires aléatoires. PhD thesis, Univ. Bordeaux 1, 1998.

[Tut63] Tutte. A census of planar maps. Canad. J. Math., 15:249-271, 1963. 